\title{
ASYMPTOTIC EXPANSIONS OF SOLUTIONS TO DIRICHLET PROBLEM FOR ELLIPTIC EQUATION WITH SINGULARITIES
}

\author{
D.A. TURSUNOV, U.Z. ERKEBAEV
}

\begin{abstract}
The paper proposes an analogue of Vishik-Lyusternik-Vasileva-Imanalieva boundary functions method for constructing a uniform asymptotic expansion of solutions to bisingular perturbed problems. By means of this method we construct the uniform asymptotic expansion for the solution to the Dirichlet problem for bisingular perturbed second order elliptic equation with two independent variables in a circle. By the maximum principle we justify formal asymptotic expansion of the solution, that is, an estimate for the error term is established.
\end{abstract}

Keywords: asymptotic expansion, Dirichlet problem, Airy function, modified Bessel functions, boundary functions.

Mathematics Subject Classification: 35J15, 35J25, 35B25, 35B40, 35C20

\section{INTRODUCTION}

Various problems for elliptic equations with a small parameter at higher derivative were studied by many authors and the literature on this issue is quite wide and well-known [1. However, problems with a double singularity, i.e., bisingularly perturbed problems, as opposed to singularly perturbed problems, are poorly studied. In bisingularly perturbed problems one singularity is related with a singular dependence of the solution on a small parameter, while the other comes from non-smooth terms in its asymptotics. In order to construct asymptotic expansions for solutions to bisingularly perturbed problems, the method of matching asymptotic expansions or Lomov regularization method is mostly used, since the classical boundary layer method can not be applied directly. In the work we propose an analogue of the classical VishikLyusternik-Vasilieva-Imanaliev boundary layer method for constructing a uniform asymptotic expansion for the solutions of bisingularly perturbed problems. By means of this method we construct a uniform asymptotic expansion of the solution to the Dirichlet problem for a bisingularly perturbed elliptic second order equation with two independent variables in a circle. In the justification of the formal asymptotic expansion of solution (FAES) we apply a maximum principle. Similar problems by this method were studied in works [3]- [5]

\section{Formulation of the PROBlem}

We study the Dirichlet problem

$$
\begin{aligned}
& \varepsilon \Delta u(\rho, \varphi, \varepsilon)-(1-\rho)(\rho-\alpha)^{2} u(\rho, \varphi, \varepsilon)=f(\rho, \varphi, \varepsilon), \quad(\rho, \varphi) \in D, \\
& u(1, \varphi, \varepsilon)=\psi(\varphi, \varepsilon),
\end{aligned}
$$

D.A. Tursunov, U.Z. Erkebaev, Asymptotic expansions of solutions to Dirichlet problem FOR ELLIPTIC EQUATION WITH SINGULARITIES.

(c) Tursunov D.A., Erkebaev U.Z. 2016.

The work is supported by MES KR.

Submitted May 25, 2015. 
where $0<\varepsilon$ is a small parameter, $\Delta=\frac{\partial^{2}}{\partial \rho^{2}}+\frac{\partial}{\rho \partial \rho}+\frac{\partial^{2}}{\rho^{2} \partial \varphi^{2}}$ is the Laplace operator,

$$
\begin{aligned}
& D=\{(\rho, \varphi) \mid 0<\rho<1,0<\varphi \leqslant 2 \pi\}, f(\rho, \varphi, \varepsilon)=\sum_{k=0}^{+\infty} f_{k}(\rho, \varphi) \varepsilon^{k}, f_{k} \in C^{\infty}(\bar{D}), \\
& \psi(\varphi, \varepsilon)=\sum_{k=0}^{+\infty} \psi_{k}(\varphi) \varepsilon^{k}, \psi_{k} \in C[0,2 \pi], \alpha \in(0,1), f(\alpha, \varphi, 0) \neq 0, f(1, \varphi, 0) \neq 0,
\end{aligned}
$$

$\psi(\varphi, \varepsilon), f(\rho, \varphi, \varepsilon)$ are given functions, $u(\rho, \varphi, \varepsilon)$ is an unknown function, $\sum_{k=0}^{+\infty} f_{k}(\rho, \varphi) \varepsilon^{k}$, $\sum_{k=0}^{+\infty} \psi_{k}(\varphi) \varepsilon^{k}$ are asymptotic series in Poincaré sense.

The solution to problem (1)-(2) exists and is unique [6]. We are interested in the asymptotic behavior of the solution to problem (1)-(2) as $\varepsilon \rightarrow 0$.

The first singularity is obvious: the solution of the limiting equation as $\varepsilon=0$

$$
-(1-\rho)(\rho-\alpha)^{2} u(\rho, \varphi, 0)=f_{0}(\rho, \varphi)
$$

does not satisfy boundary condition (2). In order to show the second singularity, we consider the structure of the external expansion of solution to problem (1), which we seek as

$$
U(\rho, \varphi, \varepsilon)=\sum_{k=0}^{+\infty} \varepsilon^{k} u_{k}(\rho, \varphi), \quad \varepsilon \rightarrow 0 .
$$

Substituting (3) into (1) and equating the coefficients at the like powers of $\varepsilon$, we obtain a simple recurrent system of equations:

$$
\begin{aligned}
& -(1-\rho)(\alpha-\rho)^{2} u_{0}(\rho, \varphi)=f_{0}(\rho, \varphi), \\
& (1-\rho)(\alpha-\rho)^{2} u_{k}(\rho, \varphi)=\Delta u_{k-1}(\rho, \varphi)-f_{k}(\rho, \varphi), \quad k \in \mathbb{N} .
\end{aligned}
$$

This is why the external expansion of the solution to problem (1)-(2) is of the form:

$$
U(\rho, \varphi, \varepsilon)=\frac{1}{(1-\rho)(\alpha-\rho)^{2}}\left(F_{0}+\ldots+\frac{\varepsilon^{k}}{(1-\rho)^{3 k}(\alpha-\rho)^{4 k}} F_{k}+\ldots\right), \quad \varepsilon \rightarrow 0,
$$

where $F_{k}(\rho, \varphi)=F_{k} \in C^{\infty}(\bar{D}), k=0,1, \ldots$

We note that functions $u_{k}(\rho, \varphi)$ have increasing singularities of the form:

$$
\begin{aligned}
& u_{k}(\rho, \varphi)=O\left(\frac{1}{(1-\rho)^{1+3 k}}\right), \quad \rho \rightarrow 1, \quad k=0,1, \ldots \\
& u_{k}(\rho, \varphi)=O\left(\frac{1}{(\rho-\alpha)^{2+4 k}}\right), \quad \rho \rightarrow \alpha, \quad k=0,1, \ldots
\end{aligned}
$$

Therefore, the considered problem is bisingularly perturbed in the terminology by A.M. Il'in [1, 2 .

\section{MAIN RESUlT}

Theorem 3.1. As $\varepsilon \rightarrow 0$, the solution of problem (1)-(2) has the asymptotic expansion:

$$
u(\rho, \varphi, \varepsilon)=\sum_{k=0}^{+\infty} \varepsilon^{k} v_{k}(\rho, \varphi)+\chi_{1}(\rho) \sum_{k=-1}^{+\infty} \varepsilon^{k / 3} w_{k}\left(\frac{1-\rho}{\varepsilon^{1 / 3}}, \varphi\right)+\chi_{2}(\rho) \sum_{k=-2}^{+\infty} \varepsilon^{k / 4} q_{k}\left(\frac{\rho-\alpha}{\varepsilon^{1 / 4}}, \varphi\right),
$$

where functions $v_{k}(\rho, \varphi), w_{k}\left(\frac{1-\rho}{\varepsilon^{1 / 3}}, \varphi\right), q_{k}\left(\frac{\rho-\alpha}{\varepsilon^{1 / 4}}, \varphi\right)$ are determined below, $\chi_{1}(\rho), \chi_{2}(\rho)$ are cut-off functions with values in $[0,1], \chi_{1}, \chi_{2} \in C^{\infty}[0,1]$, and

$$
\begin{aligned}
& \chi_{1}(\rho)=1, \quad \text { as } \quad 1-\delta \leqslant \rho \leqslant 1, \quad \text { and } \quad \chi_{1}(\rho)=0 \text { as } \quad 0 \leqslant \rho \leqslant 1-2 \delta \text {, } \\
& \chi_{2}(\rho)=1, \quad \text { as } \quad|\rho-\alpha| \leqslant \delta, \quad \text { and } \quad \chi_{2}(\rho)=0 \text { as } \quad 2 \delta \leqslant|\rho-\alpha| \text {, }
\end{aligned}
$$


$\delta \in(0, \min \{\alpha / 2,(1-\alpha) / 2\})$ is a sufficiently small number independent of $\varepsilon$.

The proof consists of two parts: construction of FAES (4) and the justification of this expansion.

3.1. Construction of FAES. We seek FAES as

$$
u(\rho, \varphi, \varepsilon)=\sum_{k=0}^{+\infty} \varepsilon^{k} v_{k}(\rho, \varphi)+\chi_{1}(\rho) \sum_{k=-1}^{+\infty} \mu^{k} w_{k}(\tau, \varphi)+\chi_{2}(\rho) \sum_{k=-2}^{+\infty} \lambda^{k} q_{k}(\eta, \varphi), \varepsilon \rightarrow 0,
$$

where $\tau=(1-\rho) / \mu, \mu=\varepsilon^{1 / 3}, \eta=(\rho-\alpha) / \lambda, \lambda=\varepsilon^{1 / 4}$.

Substituting (5) into (1), we obtain:

$$
\begin{gathered}
\sum_{k=0}^{+\infty} \varepsilon^{k}\left(\varepsilon \Delta v_{k}(\rho, \varphi)+\varepsilon \widetilde{v}_{k}(\rho, \varphi)-(1-\rho)(\alpha-\rho)^{2} v_{k}(\rho, \varphi)\right)=\sum_{k=0}^{+\infty} \varepsilon^{k}\left(f_{k}(\rho, \varphi)-h_{k}(\rho, \varphi)\right) \\
\sum_{k=-1}^{+\infty} \mu^{k+1}\left(\frac{\partial^{2} w_{k}}{\partial \tau^{2}}-\frac{\mu}{(1-\mu \tau)} \frac{\partial w_{k}}{\partial \tau}+\frac{\mu^{2}}{(1-\mu \tau)^{2}} \frac{\partial^{2} w_{k}}{\partial \varphi^{2}}-\tau(1-\alpha-\mu \tau)^{2} w_{k}\right) \\
=\sum_{k=0}^{+\infty} h_{1, k}(\tau \mu, \varphi) \mu^{3 k} \\
\sum_{k=-2}^{+\infty} \lambda^{k+2}\left(\frac{\partial^{2} q_{k}}{\partial \eta^{2}}+\frac{\lambda}{(\alpha+\lambda \eta)} \frac{\partial q_{k}}{\partial \eta}+\frac{\lambda^{2}}{(\alpha+\lambda \eta)^{2}} \frac{\partial^{2} q_{k}}{\partial \varphi^{2}}-\eta^{2}(1-\alpha-\lambda \eta) q_{k}\right) \\
=\sum_{k=0}^{+\infty} h_{2, k}(\eta \lambda, \varphi) \lambda^{4 k}
\end{gathered}
$$

The idea of our method is to introduce an unknown asymptotic series

$$
\sum_{k=0}^{+\infty} \varepsilon^{k} h_{k}(\rho, \varphi)=\chi_{1}(\rho) \sum_{k=0}^{+\infty} \varepsilon^{k} h_{1, k}(\rho, \varphi)+\chi_{2}(\rho) \sum_{k=0}^{+\infty} \varepsilon^{k} h_{2, k}(\rho, \varphi)
$$

in identities (6), (7), (8), while functions $\widetilde{v}_{k}(\rho, \varphi)$ in identity (6) are of the form:

$$
\begin{aligned}
& \widetilde{v}_{k}(\rho, \varphi)=\widetilde{w}_{k}(\rho, \varphi) \widetilde{\chi}_{1}(\rho)+2 \frac{\partial \widetilde{w}_{k}(\rho, \varphi)}{\partial \rho} \chi_{1}^{\prime}(\rho)+\widetilde{q}_{k}(\rho, \varphi) \widetilde{\chi}_{2}(\rho)+2 \frac{\partial \widetilde{q}_{k}(\rho, \varphi)}{\partial \rho} \chi_{2}^{\prime}(\rho), \\
& \widetilde{w}_{k}(\rho, \varphi)=\sum_{j=0}^{3 k} \frac{w_{-1+j, 3 k+1-j}(\varphi)}{(1-\rho)^{3 k+1-j}}, \quad \widetilde{q}_{k}(\rho, \varphi)=\sum_{j=0}^{4 k} \frac{q_{-2+j, 4 k+2-j}(\varphi)}{(\rho-\alpha)^{4 k+2-j}}, \quad \widetilde{\chi}_{j}(\rho)=\chi_{j}^{\prime \prime}(\rho)+\frac{\chi_{j}^{\prime}(\rho)}{\rho},
\end{aligned}
$$

and functions $w_{j, k}(\varphi), q_{j, k}(\varphi) \in C^{\infty}[0,2 \pi]$ are determined by the asymptotic expansions:

$$
\begin{aligned}
w_{3 k-m}(\tau, \varphi)=\sum_{j=0}^{+\infty} \frac{w_{3 k-m, 3 j+m}(\varphi)}{\tau^{3 j+m}}, & m=1,2,3 ; \quad \tau \rightarrow+\infty, \\
q_{4 k-m}(\eta, \varphi)=\sum_{j=0}^{+\infty} \frac{q_{4 k-m, 4 j+m}(\varphi)}{\eta^{4 j+m}}, & m=1,2,3,4 ; \quad k=0,1, \ldots \eta \rightarrow \pm \infty .
\end{aligned}
$$

The validity of these asymptotic expansions is proved in what follows.

To determine functions $v_{k}(\rho, \varphi)$, by identity (6) we obtain the following equations:

$$
\begin{aligned}
& -(1-\rho)(\rho-\alpha)^{2} v_{0}(\rho, \varphi)=f_{0}(\rho, \varphi)-h_{0}(\rho, \varphi), \\
& \quad \Delta v_{k-1}(\rho, \varphi)+\widetilde{v}_{k-1}(\rho, \varphi)-(1-\rho)(\rho-\alpha)^{2} v_{k}(\rho, \varphi)=f_{k}(\rho, \varphi)-h_{k}(\rho, \varphi), \quad k=1,2, \ldots
\end{aligned}
$$


This yields

$$
v_{k}(\rho, \varphi)=\frac{f_{k}(\rho, \varphi)-\Delta v_{k-1}(\rho, \varphi)-h_{k}(\rho, \varphi)}{(\rho-1)(\rho-\alpha)^{2}}+\frac{\widetilde{v}_{k-1}(\rho, \varphi)}{(1-\rho)(\rho-\alpha)^{2}}, \quad k=0,1, \ldots,
$$

and $v_{-1}(\rho, \varphi) \equiv 0, \widetilde{v}_{-1}(\rho, \varphi) \equiv 0$.

Let us determine unknown functions, that is, coefficients $h_{k}(\rho, \varphi)$ of the asymptotic series so that

$$
v_{k}(\rho, \varphi) \in C^{\infty}(\bar{D}), \quad w_{k}(\tau, \varphi) \rightarrow 0 \quad \text { as } \quad \tau \rightarrow+\infty, \quad q_{k}(\eta, \varphi) \rightarrow 0 \quad \text { as } \quad \eta \rightarrow \pm \infty .
$$

Let $g_{k}(\rho, \varphi)=f_{k}(\rho, \varphi)-\Delta v_{k-1}(\rho, \varphi)$, then $v_{k}(\rho, \varphi) \in C^{\infty}(\bar{D})$ as

$$
h_{k}(\rho, \varphi)=\chi_{1}(\rho) h_{1, k}(\rho, \varphi)+\chi_{2}(\rho) h_{2, k}(\rho, \varphi) \text {, }
$$

where

$$
\begin{aligned}
& h_{2, k}(\rho, \varphi)=g_{k, 0}(\varphi)+g_{k, 1}(\varphi)(\rho-\alpha)-\left(\frac{\rho-\alpha}{1-\alpha}\right)^{2}\left(g_{k, 0}(\varphi)+g_{k, 1}(\varphi)(1-\alpha)\right), \\
& h_{1, k}(\rho, \varphi)=\left(\frac{\rho-\alpha}{1-\alpha}\right)^{2} g_{k}(1, \varphi), \quad g_{k, 0}(\varphi)=g_{k}(\alpha, \varphi), \quad g_{k, 1}(\varphi)=\frac{\partial g_{k}(\alpha, \varphi)}{\partial \rho} .
\end{aligned}
$$

Thus, we have determined the coefficients of the series

$$
\sum_{k=0}^{+\infty} \varepsilon^{k} v_{k}(\rho, \varphi), \quad \sum_{k=0}^{+\infty} \varepsilon^{k} h_{k}(\rho, \varphi) .
$$

We proceed to determining the terms of the asymptotic series $\sum_{k=-1}^{+\infty} \mu^{k} w_{k}(\tau, \varphi)$. We write identity (7) as

$$
\begin{gathered}
\sum_{k=0}^{+\infty} \mu^{k}\left(\frac{\partial^{2} w_{-1+k}}{\partial \tau^{2}}-\mu \frac{\partial w_{-1+k}}{\partial \tau}+\mu^{2} \frac{\partial^{2} w_{-1+k}}{\partial \varphi^{2}}-\tau(1-\alpha-\mu \tau)^{2} w_{-1+k}\right) \\
=\sum_{k=0}^{+\infty} \mu^{3 k}\left(1-\frac{2 \mu \tau}{1-\alpha}+\frac{(\mu \tau)^{2}}{(1-\alpha)^{2}}\right) g_{k}(1, \varphi) .
\end{gathered}
$$

This implies:

$$
\begin{aligned}
& L w_{-1} \equiv \frac{\partial^{2} w_{-1}}{\partial \tau^{2}}-\tau(1-\alpha)^{2} w_{-1}=g_{0}(1, \varphi) \\
& L w_{0}=-2(1-\alpha) \tau^{2} w_{-1}-\frac{2 \tau}{1-\alpha} g_{0}(1, \varphi)+\frac{\partial w_{-1}}{\partial \tau} \\
& L w_{1}=-2(1-\alpha) \tau^{2} w_{0}+\tau^{3} w_{-1}+\frac{\tau^{2}}{(1-\alpha)^{2}} g_{0}(1, \varphi)+W_{1}, \\
& L w_{3 k-1}=-2(1-\alpha) \tau^{2} w_{3 k-2}+\tau^{3} w_{3 k-3}+g_{k}(1, \varphi)+W_{3 k-1}+B_{k, 0}(\varphi) \\
& L w_{3 k}=-2(1-\alpha) \tau^{2} w_{3 k-1}+\tau^{3} w_{3 k-2}-\frac{2 \tau}{1-\alpha} g_{k}(1, \varphi)+W_{3 k}+B_{k, 1}(\varphi) \tau \\
& L w_{3 k+1}=-2(1-\alpha) \tau^{2} w_{3 k}+\tau^{3} w_{3 k-1}+\frac{\tau^{2}}{(1-\alpha)^{2}} g_{k}(1, \varphi)+W_{3 k+1}+B_{k, 2}(\varphi) \tau^{2},
\end{aligned}
$$

where

$$
(\tau, \varphi) \in D_{1}=\{(\tau, \varphi) \mid 0<\tau<+\infty, 0 \leqslant \varphi<2 \pi\}, \quad W_{s}=\frac{\partial w_{s-1}}{\partial \tau}-\frac{\partial^{2} w_{s-2}}{\partial \varphi^{2}}
$$

and $B_{k, 0}(\varphi), B_{k, 1}(\varphi), B_{k, 2}(\varphi)$ are unknown functions to be determined, $k=1,2, \ldots$

Boundary conditions become

$$
w_{3 k}(0, \varphi)=\psi_{k}(\varphi)-v_{k}(1, \varphi), \quad k=0,1, \ldots, \quad w_{s}(0, \varphi)=0, \quad s \neq 3 k .
$$


Let us prove the following lemma.

Lemma 3.1. Let $\tilde{f}(\tau) \delta(\varphi) \in C^{\infty}\left(D_{1}\right), a_{0}>0$. Then the problem

$$
\frac{\partial^{2} z(\tau, \varphi)}{\partial \tau^{2}}-\tau a_{0} z(\tau, \varphi)=\tilde{f}(\tau) \delta(\varphi), \quad(\tau, \varphi) \in D_{1}, \quad z(0, \varphi)=z^{0}(\varphi)
$$

has the unique solution $z(\tau, \varphi) \in C^{\infty}\left(D_{1}\right)$.

Proof. Let $t=\sqrt[3]{a_{0}} \tau$, then problem (16) casts into the form:

$$
\frac{\partial^{2} z(t, \varphi)}{\partial t^{2}}-t z(t, \varphi)=\frac{1}{\sqrt[3]{a_{0}^{2}}} \tilde{f}(t) \delta(\varphi), \quad z(0, \varphi)=z^{0}(\varphi)
$$

We seek the a solution to problem $(17)$ as

$$
z(t, \varphi)=z_{1}(t) \frac{1}{\sqrt[3]{a_{0}^{2}}} \delta(\varphi)
$$

For $z_{1}(t)$ we obtain the problem

$$
z_{1}^{\prime \prime}(t)-t z_{1}(t)=\widetilde{f}(t), \quad z_{1}(0)=z^{0}(\varphi) \sqrt[3]{a_{0}^{2}} / \delta(\varphi) \equiv z_{1}^{0}
$$

The associated homogeneous equation

$$
z_{1}^{\prime \prime}(t)-t z_{1}(t)=0
$$

has two independent solutions $A i(t), B i(t)$, which are Airy functions [7]. By means of Airy functions we write the solution to problem $(18)$ :

$$
\begin{aligned}
z_{1}(t)= & \frac{z_{1}^{0}}{A i(0)} A i(t)+\pi B i(t) \int_{t}^{+\infty} \operatorname{Ai}(s) \widetilde{f}(s) d s \\
& +\pi A i(t)\left(\int_{0}^{t} B i(s) \widetilde{f}(s) d s-\sqrt{3} \int_{0}^{+\infty} A i(s) \widetilde{f}(s) d s\right) .
\end{aligned}
$$

This yields

$$
\begin{aligned}
z(t, \varphi)= & \frac{z^{0}(\varphi)}{A i(0)} A i(t)+\frac{\pi}{\sqrt[3]{a_{0}^{2}}} \delta(\varphi) B i(t) \int_{t}^{+\infty} A i(s) \widetilde{f}(s) d s \\
& +\frac{\pi}{\sqrt[3]{a_{0}^{2}}} \delta(\varphi) A i(t)\left(\int_{0}^{t} B i(s) \widetilde{f}(s) d s-\sqrt{3} \int_{0}^{+\infty} A i(s) \widetilde{f}(s) d s\right) .
\end{aligned}
$$

Corollary 1. If $\widetilde{f}(\tau)=O\left(\tau^{N_{1}}\right)$ as $\tau \rightarrow+\infty$, then thanks to the asymptotic behavior of Airy function as $\tau \rightarrow+\infty$, we obtain $z(\tau, \varphi)=O\left(\tau^{N_{1}-1}\right), N_{1}$ is a constant.

By means of the above lemma we prove the existence of the unique solutions to equations (9)(14) satisfying conditions (15). In what follows we shall prove the existence of functions $B_{k, 0}(\varphi)$, $B_{k, 1}(\varphi) B_{k, 2}(\varphi)$, for which the solutions to these problems belong to the class of functions with a power decay in $\tau$ and the solution to each equation (9)-(14) satisfies the identity:

$$
\lim _{\tau \rightarrow+\infty} w_{k}(\tau, \varphi)=0, \quad k=-1,0,1, \ldots
$$

We proceed to determining the terms of the asymptotic series $\sum_{k=-2}^{+\infty} \lambda^{k} q_{k}(\eta, \varphi)$. We write identity (8) as

$$
\sum_{k=0}^{+\infty} \lambda^{k}\left(\frac{\partial^{2} q_{-2+k}}{\partial \eta^{2}}+\lambda \frac{\partial q_{-2+k}}{\partial \eta}+\lambda^{2} \frac{\partial^{2} q_{-2+k}}{\partial \varphi^{2}}-\eta^{2}(1-\alpha-\lambda \eta) q_{-2+k}\right)
$$


It follows that

$$
=\sum_{k=0}^{+\infty} \lambda^{4 k}\left(g_{k, 0}(\varphi)+g_{k, 1}(\varphi) \lambda \eta-\left(\frac{\lambda \eta}{1-\alpha}\right)^{2}\left(g_{k, 0}(\varphi)+g_{k, 1}(\varphi)(1-\alpha)\right)\right) .
$$

$$
\begin{aligned}
& l q_{-2} \equiv \frac{\partial^{2} q_{-2}}{\partial \eta^{2}}-\eta^{2}(1-\alpha) q_{-2}=g_{0,0}(\varphi) \\
& l q_{-1}=-\frac{\partial q_{-2}}{\partial \eta}-\eta^{3} q_{-2}+\eta g_{0,1}(\varphi) \\
& l q_{0}=Q_{0}-\eta^{3} q_{-1}-\left(\frac{\eta}{1-\alpha}\right)^{2}\left(g_{0,0}(\varphi)+g_{0,1}(\varphi)(1-\alpha)\right), \\
& l q_{1}=Q_{1}-\eta^{3} q_{0}, \\
& l q_{4 k-2}=Q_{4 k-2}-\eta^{3} q_{4 k-3}+g_{k, 0}(\varphi)+A_{k, 0}(\varphi), \\
& l q_{4 k-1}=Q_{4 k-1}-\eta^{3} q_{4 k-2}+\eta g_{k, 1}(\varphi)+A_{k, 1}(\varphi) \eta \\
& l q_{4 k}=Q_{4 k}-\eta^{3} q_{4 k-1}-\left(\frac{\eta}{1-\alpha}\right)^{2}\left(g_{k, 0}(\varphi)+g_{k, 1}(\varphi)(1-\alpha)\right)+A_{k, 2}(\varphi) \eta^{2} \\
& l q_{4 k+1}=Q_{4 k+1}-\eta^{3} q_{4 k},
\end{aligned}
$$

where

$$
(\eta, \varphi) \in D_{2}=\{(\eta, \varphi) \mid-\infty<\eta<+\infty, 0 \leqslant \varphi<2 \pi\}, \quad Q_{s}=-\frac{\partial q_{s-1}}{\partial \eta}-\frac{\partial^{2} q_{s-2}}{\partial \varphi^{2}}
$$

and $A_{k, 0}(\varphi), A_{k, 1}(\varphi), A_{k, 2}(\varphi)$ are unknown functions to be determined, $k=1,2, \ldots$

Let us prove the following auxiliary lemma implying the existence of solutions to equations (19) $-(26)$.

Lemma 3.2. Let $\widetilde{f}(\eta) \delta(\varphi) \in C^{\infty}\left(D_{2}\right), b_{0}>0$. Then the problem

$$
\frac{\partial^{2} z(\eta, \varphi)}{\partial \eta^{2}}-\eta^{2} b_{0} z(\eta, \varphi)=\tilde{f}(\eta) \delta(\varphi), \quad(\eta, \varphi) \in D_{2},
$$

has the unique solution $z(\eta, \varphi) \in C^{\infty}\left(D_{2}\right)$.

Proof. By the change $\eta=\sqrt[4]{4 / b_{0}} t$ we obtain the equation

$$
\frac{\partial^{2} z(t, \varphi)}{\partial t^{2}}-t^{2} z(t, \varphi)=\frac{2}{\sqrt{b_{0}}} \tilde{f}(t) \delta(\varphi) .
$$

We seek the solution to this equation as

$$
z(t, \varphi)=z_{2}(t) \sqrt{\frac{4}{b_{0}}} \delta(\varphi)
$$

Then for $z_{2}(t)$ we obtain the equation:

$$
z_{2}^{\prime \prime}(t)-t^{2} z_{2}(t)=\tilde{f}(t)
$$

and the associated homogeneous equation

$$
z_{2}^{\prime \prime}(t)-t^{2} z_{2}(t)=0
$$

has a fundamental system of solutions $\left\{U_{4}(t), U_{4}(-t)\right\}$, where $U_{4}(t)=\sqrt{\frac{2 t}{\pi}} K_{1 / 4}\left(t^{2}\right), t>0$, $K_{1 / 4}\left(t^{2}\right)$ is the McDonald function (modified Bessel function) [7]. Let us describe the basic properties of these functions $U_{4}(t), U_{4}(-t)$ :

a) The Wronskian of these functions is equal to

$$
W\left(U_{4}(t), U_{4}(-t)\right)=4 \csc (\pi / 4)=4 \sqrt{2} .
$$


b) As $t=0, U_{4}(0)=\pi^{-1 / 2} 2^{-1 / 4} \Gamma(1 / 4)$.

c) As $t \rightarrow+\infty$, function $U_{4}(t)$ decays exponentially: $U_{4}(t) \sim t^{-1 / 2} e^{-t^{2}}$.

As $t \rightarrow-\infty$, the function $U_{4}(t)=\sqrt{\frac{2|t|}{\pi}}\left(\sqrt{2} \pi I_{1 / 4}\left(t^{2}\right)+K_{1 / 4}\left(t^{2}\right)\right), t<0$, grows exponentially:

$$
U_{4}(t)=(2 / t)^{1 / 2} e^{t^{2}}\left(1+O\left(t^{-2}\right)\right),
$$

where $I_{1 / 4}\left(t^{2}\right), K_{1 / 4}\left(t^{2}\right)$ are modified Bessel functions. Therefore, the solution to problem (27) can be written as

$$
z(t, \varphi)=\frac{1}{2 \sqrt{2 b_{0}}} \delta(\varphi)\left(U_{4}(t) \int_{-\infty}^{t} U_{4}(-s) \widetilde{f}(s) d s+U_{4}(-t) \int_{t}^{+\infty} U_{4}(s) \widetilde{f}(s) d s\right),
$$

where $t=\sqrt[4]{b_{0} / 4} \eta$.

Corollary 2. If $\tilde{f}(\eta)=O\left(\eta^{N_{2}}\right)$ as $\eta \rightarrow \pm \infty$, then $z(\eta, \varphi)=O\left(\eta^{N_{2}-2}\right), N_{2}$ is a constant.

Employing this lemma, we can write explicitly the solutions to problems $(19)-(26)$.

Let us prove the existence of functions $A_{k, 0}(\varphi), A_{k, 1}(\varphi), A_{k, 2}(\varphi)$, for which the identities

$$
\lim _{\eta \rightarrow \pm \infty} q_{k}(\eta, \varphi)=0, \quad k=-2,-1,0,1, \ldots
$$

hold true.

Lemma 3.3. There exist functions $A_{k, j}(\varphi), B_{k, j}(\varphi) \in C^{\infty}[0,2 \pi], k \in N, j=0,1,2$, satisfying the identities $\left(A_{k, j}=A_{k, j}(\varphi), B_{k, j}=B_{k, j}(\varphi)\right)$ :

$$
\begin{aligned}
& A_{k, 0}-\alpha A_{k, 1}+\alpha^{2} A_{k, 2}+B_{k, 0}+B_{k, 1}+B_{k, 2}=0, \\
& A_{k, 1}-2 \alpha A_{k, 2}-B_{k, 1}-2 B_{k, 2}=0 \\
& A_{k, 2}+B_{k, 2}=0
\end{aligned}
$$

and the relations

$$
\begin{array}{llll}
w_{3 k-m}(\tau, \varphi)=\sum_{j=0}^{\infty} \frac{w_{3 k-m, 3 j+m}(\varphi)}{\tau^{3 j+m}}, \quad m=1,2,3, \quad w_{k, j} \in C^{\infty}[0,2 \pi], & \tau \rightarrow+\infty, \\
q_{4 k-m}(\eta, \varphi)=\sum_{j=0}^{\infty} \frac{q_{4 k-m, 4 j+m}(\varphi)}{\eta^{4 j+m}}, & m=1,2,3,4, \quad q_{k, j} \in C^{\infty}[0,2 \pi], & \eta \rightarrow \pm \infty .
\end{array}
$$

hold true.

Proof. The meaning of identities (28)-(30) is that such choice of functions keeps the smoothness of solutions $v_{k}(\rho, \varphi)$, i.e.,

$$
A_{k, 0}+A_{k, 1}(\rho-\alpha)+A_{k, 2}(\rho-\alpha)^{2}+B_{k, 0}+B_{k, 1}(1-\rho)+B_{k, 2}(1-\rho)^{2} \equiv 0 .
$$

We observe that as $\tau \rightarrow+\infty$ and $\eta \rightarrow \pm \infty$, the relations

$$
\begin{array}{ll}
w_{-1}(\tau, \varphi)=\sum_{j=0}^{\infty} \frac{w_{-1,3 j+1}(\varphi)}{\tau^{3 j+1}}, & \\
w_{m}(\tau, \varphi)=\sum_{j=1}^{\infty} \frac{w_{m, 3 j-m}(\varphi)}{\tau^{3 j-m}}, & m=0,1, \\
q_{-m}(\eta, \varphi)=\sum_{j=0}^{\infty} \frac{q_{-m, 4 j+m}(\varphi)}{\eta^{4 j+m}}, & m=2,1, \\
q_{m}(\eta, \varphi)=\sum_{j=1}^{\infty} \frac{q_{m, 4 j-m}(\varphi)}{\eta^{4 j-m}}, & m=0,1,
\end{array}
$$


hold true, where $w_{k, j}(\varphi), q_{k, j}(\varphi) \in C^{\infty}[0,2 \pi]$.

Suppose that as $\tau \rightarrow+\infty$ and $\eta \rightarrow \pm \infty$ for some $k=0,1, \ldots$, the relations

$$
\begin{array}{ll}
w_{3 k-1}(\tau, \varphi)=\sum_{j=0}^{\infty} \frac{w_{3 k-1,3 j+1}(\varphi)}{\tau^{3 j+1}}, & \\
w_{3 k+m}(\tau, \varphi)=\sum_{j=1}^{\infty} \frac{w_{3 k+m, 3 j-m}(\varphi)}{\tau^{3 j-m}}, & m=0,1, \\
q_{4 k-m}(\eta, \varphi)=\sum_{j=0}^{\infty} \frac{q_{4 k-m, 4 j+m}(\varphi)}{\eta^{4 j+m}}, & m=2,1, \\
q_{4 k+m}(\eta, \varphi)=\sum_{j=1}^{\infty} \frac{q_{4 k+m, 4 j-m}(\varphi)}{\eta^{4 j-m}}, & m=0,1,
\end{array}
$$

hold true, where $w_{k, j}, q_{k, j} \in C^{\infty}[0,2 \pi]$. Then for $k+1$ we have

$$
\begin{aligned}
& L w_{3 k+2}=-2(1-\alpha) \tau^{2} w_{3 k+1}+\tau^{3} w_{3 k}+g_{k+1}(1, \varphi)+\frac{\partial w_{3 k+1}}{\partial \tau}-\frac{\partial^{2} w_{3 k}}{\partial \varphi^{2}}+B_{k+1,0}, \\
& L w_{3 k+3}=-2(1-\alpha) \tau^{2} w_{3 k+2}+\tau^{3} w_{3 k+1}-\frac{2 \tau}{1-\alpha} g_{k+1}(1, \varphi)+\frac{\partial w_{3 k+2}}{\partial \tau}-\frac{\partial^{2} w_{3 k+1}}{\partial \varphi^{2}}+B_{k+1,1} \tau, \\
& L w_{3 k+4}=-2(1-\alpha) \tau^{2} w_{3 k+3}+\tau^{3} w_{3 k+2}+\frac{\tau^{2}}{(1-\alpha)^{2}} g_{k+1}(1, \varphi)+\frac{\partial w_{3 k+3}}{\partial \tau}-\frac{\partial^{2} w_{3 k+2}}{\partial \varphi^{2}}+B_{k+1,2} \tau^{2}, \\
& l q_{4 k+2}=-\frac{\partial q_{4 k+1}}{\partial \eta}-\frac{\partial^{2} q_{4 k}}{\partial \varphi^{2}}-\eta^{3} q_{4 k+1}+g_{k+1,0}(\varphi)+A_{k+1,0}, \\
& l q_{4 k+3}=-\frac{\partial q_{4 k+2}}{\partial \eta}-\frac{\partial^{2} q_{4 k+1}}{\partial \varphi^{2}}-\eta^{3} q_{4 k+2}+\eta g_{k+1,1}(\varphi)+A_{k+1,1} \eta \\
& l q_{4 k+4}=-\frac{\partial q_{4 k+3}}{\partial \eta}-\frac{\partial^{2} q_{4 k+2}}{\partial \varphi^{2}}-\eta^{3} q_{4 k+3}-\left(\frac{\eta}{1-\alpha}\right)^{2}\left(g_{k+1,0}(\varphi)+g_{k+1,1}(\varphi)(1-\alpha)\right)+A_{k+1,2} \eta^{2}, \\
& l q_{4 k+5}=-\frac{\partial q_{4 k+4}}{\partial \eta}-\frac{\partial^{2} q_{4 k+3}}{\partial \varphi^{2}}-\eta^{3} q_{4 k+4} .
\end{aligned}
$$

This implies

$$
\begin{aligned}
w_{3 k+2}= & \sum_{j=0}^{\infty} \frac{w_{3 k+2,3 j+1}}{\tau^{3 j+1}}, \\
w_{3 k+3}= & \sum_{j=0}^{\infty} \frac{w_{3 k+3,3 j+3}}{\tau^{3 j+3}} \quad \text { as } \quad B_{k+1,1}=-\frac{2 B_{k+1,0}}{1-\alpha}+3 w_{3 k+1,2}-\frac{2 w_{3 k, 3}}{1-\alpha}, \\
w_{3 k+4}= & \sum_{j=0}^{\infty} \frac{w_{3 k+4,3 j+2}}{\tau^{3 j+2}} \quad \text { as } \quad B_{k+1,2}=\frac{B_{k+1,0}}{(1-\alpha)^{2}}-\frac{2 w_{3 k+1,2}}{1-\alpha}+\frac{w_{3 k, 3}}{(1-\alpha)^{2}}, \\
q_{4 k+2}= & \sum_{j=0}^{\infty} \frac{q_{4 k+2,4 j+2}}{\eta^{4 j+2}}, \quad q_{4 k+3}=\sum_{j=0}^{\infty} \frac{q_{4 k+3,4 j+1}}{\eta^{4 j+1}}, \quad q_{4 k+5}=\sum_{j=0}^{\infty} \frac{q_{4 k+5,4 j+3}}{\eta^{4 j+3}}, \\
q_{4 k+4}= & \sum_{j=0}^{\infty} \frac{q_{4 k+4,4 j+4}}{\eta^{4 j+4}} \quad \text { as } \quad A_{k+1,2}=-\frac{A_{k+1,0}}{(1-\alpha)^{2}}-\frac{A_{k+1,1}}{1-\alpha}+\frac{q_{4 k+1,3}}{(1-\alpha)^{2}},
\end{aligned}
$$


where $w_{k, j}=w_{k, j}(\varphi), q_{k, j}=q_{k, j}(\varphi)$. As a result we obtain a system of linear algebraic equations with three unknowns $A_{k+1,0}, A_{k+1,1}, B_{k+1,0}$ :

$$
\begin{aligned}
& \frac{1-2 \alpha}{(1-\alpha)^{2}} A_{k+1,0}-\frac{\alpha}{1-\alpha} A_{k+1,1}+\alpha^{2} c+c_{1}+\frac{\alpha^{2}}{(1-\alpha)^{2}} B_{k+1,0}=0, \\
& \frac{2 \alpha}{(1-\alpha)^{2}} A_{k+1,0}+\frac{1+\alpha}{1-\alpha} A_{k+1,1}-2 \alpha^{2} c-c_{1}-2 c_{2}-\frac{2 \alpha}{(1-\alpha)^{2}} B_{k+1,0}=0, \\
& \frac{1}{(1-\alpha)^{2}} A_{k+1,0}+\frac{1}{1-\alpha} A_{k+1,1}-c-c_{2}-\frac{1}{(1-\alpha)^{2}} B_{k+1,0}=0,
\end{aligned}
$$

where $c=\frac{q_{4 k+1,3}}{(1-\alpha)^{2}}, c_{1}=3 w_{3 k+1,2}-\frac{2 w_{3 k, 3}}{1-\alpha}, c_{2}=\frac{w_{3 k, 3}}{(1-\alpha)^{2}}-\frac{2 w_{3 k+1,2}}{1-\alpha}$.

The system has the unique solution

$$
\begin{aligned}
& B_{k+1,0}=-q_{4 k+1,3}+\frac{w_{3 k, 3}}{(1-\alpha)^{2}}-\frac{2 w_{3 k+1,2}}{1-\alpha} \\
& A_{k+1,1}=w_{3 k+1,2}, \quad A_{k+1,0}=w_{3 k, 3}\left(1+\frac{1}{(1-\alpha)^{2}}\right)-w_{3 k+1,2}\left(3(1-\alpha)+\frac{2}{1-\alpha}\right) .
\end{aligned}
$$

Therefore, for each $k=0,1, \ldots$, identities (31) and (32) hold true, i.e.,

$$
\lim _{\eta \rightarrow \pm \infty} q_{k-2}(\eta, \varphi)=0, \quad \lim _{\tau \rightarrow+\infty} w_{k-1}(\tau, \varphi)=0, \quad k=0,1, \ldots
$$

We observe that

$$
\begin{aligned}
& w_{3 k-m}\left(\frac{1-\rho}{\mu}, \varphi\right)=\mu^{m} \sum_{j=0}^{\infty} \varepsilon^{j} \frac{w_{3 k-m, 3 j+m}(\varphi)}{(1-\rho)^{3 j+m}}, \quad m=1,2,3, \quad \varepsilon \rightarrow 0, \\
& q_{4 k-m}\left(\frac{\rho-\alpha}{\lambda}, \varphi\right)=\lambda^{m} \sum_{j=0}^{\infty} \varepsilon^{j} \frac{q_{4 k-m, 4 j+m}(\varphi)}{(\rho-\alpha)^{4 j+m}}, \quad m=1,2,3,4, \quad k=0,1, \ldots, \quad \varepsilon \rightarrow 0 .
\end{aligned}
$$

3.2. Justification of FAES. We proceed to justifying of formal asymptotic expansion (5). Let

$$
\begin{aligned}
& u_{n}(\rho, \varphi, \varepsilon)=\sum_{k=0}^{n} \varepsilon^{k} v_{k}(\rho, \varphi)+\chi_{1}(\rho) \sum_{k=-1}^{3 n+1} \mu^{k} w_{k}(\tau, \varphi)+\chi_{2}(\rho) \sum_{k=-2}^{4 n} \lambda^{k} q_{k}(\eta, \varphi), \\
& R(\rho, \varphi, \varepsilon)=u(\rho, \varphi, \varepsilon)-u_{n}(\rho, \varphi, \varepsilon) .
\end{aligned}
$$

We note that $u_{n}(\rho, \varphi, \varepsilon) \in C^{\infty}(D), \varepsilon \rightarrow 0$.

For the residual term $R(\rho, \varphi, \varepsilon)$ we obtain the equation:

$$
\varepsilon \Delta R(\rho, \varphi, \varepsilon)-(1-\rho)(\alpha-\rho)^{2} R(\rho, \varphi, \varepsilon)=\varepsilon^{n+3 / 4} \Phi, \quad(\rho, \varphi) \in D,
$$

where

$$
\begin{aligned}
& \Phi=\left(\tilde{f}(\rho, \varphi, \varepsilon)-\Delta v_{n}(\rho, \varphi)-\widetilde{v}_{n}(\rho, \varphi, \varepsilon)+\left(\tau^{3} w_{3 n}(\tau, \varphi)-2(1-\alpha) \tau^{2} w_{3 n+1}(\tau, \varphi)\right.\right. \\
&\left.\left.+\frac{\partial w_{3 n+1}(\tau, \varphi)}{\partial \tau}-\frac{\partial^{2}\left(w_{3 n}(\tau, \varphi)+\mu w_{3 n+1}(\tau, \varphi)\right)}{\partial \varphi^{2}}+\mu \tau^{3} w_{3 n+1}(\tau, \varphi)\right) \chi_{1}(\rho)\right) \varepsilon^{1 / 4} \\
&-\left(\eta^{3} q_{4 n}(\eta, \varphi)+\frac{\partial q_{4 n}(\eta, \varphi)}{\partial \eta}+\frac{\partial^{2}\left(q_{4 n-1}(\eta, \varphi)+\lambda q_{4 n}(\eta, \varphi)\right)}{\partial \varphi^{2}}\right) \chi_{2}(\rho), \\
& \tilde{f}(\rho, \varphi, \varepsilon)=\sum_{k=0}^{\infty} \varepsilon^{k} f_{n+1+k}(\rho, \varphi), \quad \widetilde{v}_{n}(\rho, \varphi, \varepsilon)=\sum_{k=0}^{\infty} \varepsilon^{k} \widetilde{v}_{n+k}(\rho, \varphi) .
\end{aligned}
$$


We observe that $\Phi$ is a smooth function. This is as $\varepsilon \rightarrow 0$ there exists a constant $M>0$ such that $\|\Phi\|_{C} \leqslant M$, i.e., $\Phi=O(1)$. The boundary condition becomes:

$$
R(1, \varphi, \varepsilon)=\sum_{k=n+1}^{\infty} \varepsilon^{k} \psi_{k}(\varphi) \quad \text { or } \quad R(1, \varphi, \varepsilon)=O\left(\varepsilon^{n+1}\right), \quad \varepsilon \rightarrow 0 .
$$

Thus, we obtain the following problem:

$$
\begin{aligned}
& \varepsilon \Delta R(\rho, \varphi, \varepsilon)-(1-\rho)(\alpha-\rho)^{2} R(\rho, \varphi, \varepsilon)=O\left(\varepsilon^{n+3 / 4}\right), \quad \varepsilon \rightarrow 0, \quad(\rho, \varphi) \in D, \\
& R(1, \varphi, \varepsilon)=O\left(\varepsilon^{n+1}\right), \quad \varepsilon \rightarrow 0 .
\end{aligned}
$$

Applying the maximum principle to this problem, we obtain

$$
R(\rho, \varphi, \varepsilon)=O\left(\varepsilon^{n-1 / 4}\right), \quad \varepsilon \rightarrow 0 .
$$

This implies the validity of expansion (4). The proof of the theorem is complete.

Example. Let

$$
a(\rho, \varphi) \equiv 1, \quad f(\rho, \varphi)=1+\rho \cos \varphi+\rho^{2} \sin \varphi+\rho^{3}, \quad \psi(\varphi, \varepsilon) \equiv 0,
$$

that is, we study the problem

$$
\begin{aligned}
& \varepsilon \Delta u(\rho, \varphi, \varepsilon)-(1-\rho)\left(\frac{1}{2}-\rho\right)^{2} u(\rho, \varphi, \varepsilon)=1+\rho \cos \varphi+\rho^{2} \sin \varphi+\rho^{3}, \quad(\rho, \varphi) \in D, \\
& u(1, \varphi, \varepsilon)=0,
\end{aligned}
$$

then

If

$$
u_{0}(\rho, \varphi)=-\frac{1+\rho \cos \varphi+\rho^{2} \sin \varphi+\rho^{3}}{(1-\rho)\left(\frac{1}{2}-\rho\right)^{2}}
$$

$$
\begin{aligned}
h_{1,0}(\rho, \varphi)= & (2 \rho-1)^{2}(2+\cos \varphi+\sin \varphi), \\
h_{2,0}(\rho, \varphi)= & \frac{9}{8}+\frac{1}{2} \cos \varphi+\frac{1}{4} \sin \varphi+\left(\rho-\frac{1}{2}\right)\left(\frac{3}{4}+\cos \varphi+\sin \varphi\right) \\
& -\left(\rho-\frac{1}{2}\right)^{2}(6+4 \cos \varphi+3 \sin \varphi),
\end{aligned}
$$

then

$$
\begin{aligned}
& v_{0}(\rho, \varphi)=-\frac{1+\rho \cos \varphi+\rho^{2} \sin \varphi+\rho^{3}-h_{1,0}(\rho, \varphi) \chi_{1}(\rho)-h_{2,0}(\rho, \varphi) \chi_{2}(\rho)}{(1-\rho)\left(\frac{1}{2}-\rho\right)^{2}}, \\
& v_{1}(\rho, \varphi)=\frac{\Delta v_{0}(\rho, \varphi)-h_{1,1}(\rho, \varphi) \chi_{1}(\rho)-h_{2,1}(\rho, \varphi) \chi_{2}(\rho)}{(1-\rho)\left(\frac{1}{2}-\rho\right)^{2}}+\frac{\widetilde{v}_{0}(\rho, \varphi)}{(1-\rho)\left(\frac{1}{2}-\rho\right)^{2}}
\end{aligned}
$$

where

$$
\begin{aligned}
& h_{2,1}(\rho, \varphi)=g_{1,0}(\varphi)+g_{1,1}(\varphi)(\rho-1 / 2)-(2 \rho-1)^{2}\left(g_{1,0}(\varphi)+g_{1,1}(\varphi) / 2\right), \\
& h_{1,1}(\rho, \varphi)=(2 \rho-1)^{2} \Delta v_{0}(1, \varphi), \quad g_{1,0}(\varphi)=\Delta v_{0}(1 / 2, \varphi), \quad g_{1,1}(\varphi)=\left.\frac{\partial}{\partial \rho} \Delta v_{0}(\rho, \varphi)\right|_{\rho=1 / 2}, \\
& \widetilde{v}_{0}(\rho, \varphi)=\widetilde{w}_{0}(\rho, \varphi) \widetilde{\chi}_{1}(\rho)+2 \frac{\partial \widetilde{w}_{0}(\rho, \varphi)}{\partial \rho} \chi_{1}^{\prime}(\rho)+\widetilde{q}_{0}(\rho, \varphi) \widetilde{\chi}_{2}(\rho)+2 \frac{\partial \widetilde{q}_{0}(\rho, \varphi)}{\partial \rho} \chi_{2}^{\prime}(\rho), \\
& \widetilde{w}_{0}(\rho, \varphi)=-\frac{4(2+\cos \varphi+\sin \varphi)}{1-\rho}, \quad \widetilde{q}_{0}(\rho, \varphi)=-\frac{9+4 \cos \varphi+2 \sin \varphi}{(2 \rho-1)^{2}} .
\end{aligned}
$$

For $w_{k}(\tau, \varphi)$ and $q_{k}(\eta, \varphi)$ we obtain problems similar to problems (9)-(14), (15), (19)-(26). The solutions to these problems exist, are unique and as $\tau \rightarrow+\infty, \eta \rightarrow \pm \infty$, the estimates hold true:

$$
w_{-1}(\tau, \varphi)=w_{2}(\tau, \varphi)=O\left(\tau^{-1}\right), \quad w_{0}(\tau, \varphi)=w_{3}(\tau, \varphi)=O\left(\tau^{-3}\right),
$$




$$
\begin{array}{ll}
w_{1}(\tau, \varphi)=w_{4}(\tau, \varphi)=O\left(\tau^{-2}\right), & q_{-2}(\eta, \varphi)=q_{2}(\eta, \varphi)=O\left(\eta^{-2}\right), \\
q_{-1}(\eta, \varphi)=q_{3}(\eta, \varphi)=O\left(\eta^{-1}\right), & q_{0}(\eta, \varphi)=q_{4}(\eta, \varphi)=O\left(\eta^{-4}\right), \quad q_{1}(\eta, \varphi)=O\left(\eta^{-3}\right) .
\end{array}
$$

Therefore, for the solution to problem (33)-34 the expansion

$$
\begin{aligned}
u(\rho, \varphi, \varepsilon)= & v_{0}(\rho, \varphi)+\varepsilon v_{1}(\rho, \varphi)+\chi_{1}(\rho) \sum_{k=-1}^{4} \varepsilon^{k / 3} w_{k}\left(\frac{1-\rho}{\varepsilon^{1 / 3}}, \varphi\right) \\
& +\chi_{2}(\rho) \sum_{k=-2}^{4} \varepsilon^{k / 4} q_{k}\left(\frac{2 \rho-1}{2 \varepsilon^{1 / 4}}, \varphi\right)+O\left(\varepsilon^{3 / 4}\right), \quad \varepsilon \rightarrow 0,
\end{aligned}
$$

holds true.

Conclusion. The feature of the considered problem is that it is bisingular. For this case we have proved the applicability of the boundary layer method. We have constructed the uniform asymptotic expansion in the small parameter for the solution to the Dirichlet problem for a bisingularly perturbed elliptic second order differential equation with two independent variables in a circle. Moreover, the constructed asymptotic series is a Puiseux series.

The proposed method differs from the method of matching asymptotics by the fact that the increasing singularities of the external expansion are removed and by means of the series with coefficients $h_{k}$ the singularities are moved to the internal expansion.

It should be noted that for simplicity, we have studied here the case $a(\rho, \varphi) \equiv 1$. The asymptotic expansion of the solution to the Dirichlet problem for the equation

$$
\varepsilon \Delta u(\rho, \varphi, \varepsilon)-(1-\rho)(\rho-\alpha)^{2} a(\rho, \varphi) u(\rho, \varphi, \varepsilon)=f(\rho, \varphi, \varepsilon), \quad(\rho, \varphi) \in D,
$$

where $a(\rho, \varphi)>0$ in domain $\bar{D}$ can be constructed in the same way and it has the same structure.

\section{BIBLIOGRAPHY}

1. A.M. Il'in. Matching of asymptotic expansions of solutions of boundary value problems. Nauka, Moscow (1989). [Amer. Math. Soc., Providence, RI (1992).]

2. A.M. Il'in, A.R. Danilin. Asymptotic methods in analysis. Fizmatlit, Moscow (2009). (in Russian).

3. D.A. Tursunov. Asymptotic expansion of the solution of the bisingularly perturbed elliptic equation // Vestn. Tomsk. Gosud. Univ. Matem. Mekh. 6:26, 37-44 (2013). (in Russian).

4. D.A. Tursunov. Asymptotics of solution to a bisingularly perturbed elliptic equation. The case of a singular point at the boundary // Izv. Tomsk. Politech. Univ. 324:2, 31-35 (2014). (in Russian).

5. D.A. Tursunov, K.J. Belekov. Asymptotic expansion of the solution of the Dirichlet problem for bisingular perturbed elliptic equations in domains with smooth boundaries // Proceedings of V Congress of the Turkic World Mathematicians. Kyrgyzstan, Bulan-Sogottu. 143-147 (2014).

6. D. Gilbarg, N.S. Trudinger. Elliptic partial differential equations of second order. Springer, Berlin (1983).

7. M.V. Fedoryuk. Asymptotic analysis: linear ordinary differential equations. Nauka, Moscow (1977). [Springer-Verlag, Berlin (1993).]

Dilmurat Abdillazhanovich Tursunov,

Ural State Pedagogical University,

Karl Liebknecht str. 9,

620151, Ekaterinburg, Russia

E-mail: d_osh@rambler.ru

Ulukbek Zairbekovich Erkebaev,

Osh State University,

Lenin str. 331,

723500, Osh, Kyrgyzstan

E-mail: uluk3188@mail.ru 